\title{
Condiciones de habitabilidad de la vivienda sustentable de interés social. Caso "Los Héroes San Pablo II", Tecamac, Estado de México
}

\author{
Conditions of habitability of sustainable housing of social interest. Case of \\ "Los Héroes San Pablo II", Tecamac, Estado of Mexico
}

\author{
José Juan Méndez-Ramírez* \\ Teresa Becerril-Sánchez* \\ Juan José Gutiérrez-Chaparro*
}

Recibido: enero 14 de 2020.

Aceptado: enero 27 de 2021.

\begin{abstract}
Resumen
El objetivo de este trabajo es analizar si la vivienda sustentable de interés social, promovida por INFONAVIT a través de su programa Hipoteca verde, presenta condiciones óptimas de habitabilidad y cumple con los principios de sustentabilidad; para ello, se tomó como objeto de estudio el conjunto urbano “Los Héroes San Pablo II” Tecamac, Estado de México.

Para llevar a cabo la investigación, se empleó información documental a partir de la cual se definieron los fundamentos conceptuales; se revisaron los informes anuales que el INFONAVIT presenta en línea; se desarrolló trabajo de campo aplicando entrevistas semi estructuradas a residentes de este conjunto urbano, así como una lista de comprobación; y, finalmente, se recurrió a la técnica de observación.

Con esta investigación se constató que la sustentabilidad de la vivienda considera variables principalmente energéticas, las cuales tienen repercusiones positivas en la economía de los habitantes, sobre todo por el uso de ecotecnologías; sin embargo, pone poca atención en el aspecto social. En relación con el aspecto medio ambiental, la vivienda no cuenta con infraestructura que separe aguas grises de residuales ni se da un adecuado manejo de residuos sólidos, entre otros. La habitabilidad de estas viviendas no es la óptima; presenta problemas de hacinamiento provocando falta de privacidad y problemas familiares; además, por estar ubicadas un tanto alejadas del equipamiento urbano, se genera un elevado número de traslados entre los residentes, lo que contribuye a crear situaciones de sustentabilidad.
\end{abstract}

Palabras clave: vivienda sustentable, habitabilidad, ecotecnologías en la vivienda.

\begin{abstract}
The objective of this paper is to analyze sustainable housing, promoted by INFONAVIT through its program "Hipoteca Verde", it presents optimal habitability conditions, and complies with the principles of sustainability, for this purpose the set was taken as an urban object of study "Los Héroes San Pablo II" Tecamac, state of Mexico. To develop the research, documentary information was used from which the conceptual foundations were defined, as well as the review of the annual reports that INFONAVIT has online; fieldwork was developed, applying semi-structured interviews to residents of this urban complex, as well as a checklist and observation.

As a result, it was found that the sustainability of housing focuses mainly on alternatives for energy that have positive repercussions on the economy of the inhabitants, mainly due to the use of eco-technologies, despite the little attention given to the social aspect. In the environmental dimension, the house does not have infrastructure that separates dirty water from residuals, adding the bad management of solid waste. The habitability in this type of housing is not optimal due to overcrowding problems, causing lack of privacy and family problems, because they are far from urban facilities, it has repercussions in a high number of transfers by residents, creating in this way, unsustainable situations derived from the quality of construction materials and the house deteriorates in a short time.
\end{abstract}

Keywords: Sustainable housing, habitability, eco-technologies in housing.

*Universidad Autónoma del Estado de México, México. Correos electrónicos: cidfino@yahoo.com, tebecerril_3@yahoo.com.mx, urbania_jj@hotmail.com 


\section{Introducción}

La vivienda es uno de los grandes temas nacionales que ha sido objeto de estudio de diversas investigaciones, y en la actualidad se ha complicado aún más al acompañarla con el adjetivo sustentable. La vivienda y su problema se han explicado desde distintas disciplinas y enfoques teóricos; algunos han resaltado la producción de la vivienda enfatizando el papel de los actores económicos (De Mattos, 2015; Scheteingart y García, 2006, citado en Cámara de Diputados del H. Congreso de la Unión, CESOP (2006); otros han buscado comprender el proceso de ocupación del suelo no sólo por los sectores marginados y pobres, sino también por los grupos de capital inmobiliario que han hecho de la periferia urbana un espacio de atracción para los sectores medios y altos (Zulaica y Celemín, 2008); hay quienes se han ocupado del estudio de la segregación residencial (Montejano et al., 2018); otros más han destacado situaciones psico-sociales derivadas de problemas de hacinamiento (Lentini y Palero, 1997); o quienes han tomado a la política urbana como objeto de análisis (Ziccardi y González, 2013; Maycotte y Sánchez, 2009), entre otras.

De ahí se establece como objetivo analizar si la vivienda sustentable de interés social, promovida por el Fondo Nacional de la Vivienda para los Trabajadores (INFONAVIT) a través de su programa Hipoteca Verde, presenta condiciones óptimas de habitabilidad y cumple con los principios de sustentabilidad; para ello, se tomó como objeto de estudio el conjunto urbano “Los Héroes San Pablo II" Tecamac, Estado de México.

Las investigaciones realizadas en torno a la vivienda y a la habitabilidad se han realizado desde distintas miradas disciplinarias; por ejemplo, Narbondo (2014) estudia la transformación de la política pública y de vivienda vinculada al cambio de Estado Benefactor a Estado Neoliberal; Coulomb y Schteingart (2006) analizan los efectos de las reformas al artículo 115 constitucional en la ocupación del suelo y la producción de vivienda por parte de los grupos inmobiliarios privados; Eibenschutz y Goya (2009) toman como objeto de estudio los problemas de habitabilidad que presentan las viviendas construidas en la periferia de las ciudades; situación que enfrenta a sus residentes a la carencia de servicios, comunicación, movilidad, equipamiento, entre otros; por otro lado, Maya (1999) ha analizado la vivienda de interés social a nivel metropolitano.

Para el caso de la habitabilidad, los análisis desarrollados giran en torno a preguntas que buscan saber cómo es la habitabilidad en las viviendas de interés social, tal como lo manifestaron Molar y Aguirre (2013); asimismo, Orozco y Guzmán (2015) destacan el problema de la gestión ambiental metropolitana vinculado al sistema habitacional; Moreno (2008) destaca la condición de habitabilidad que presentan las viviendas como una condición de calidad de vida; Mena (2011) relaciona a la habitabilidad de la vivienda de interés social con la cultura, como un elemento importante de las condiciones de vida. 
Por su parte, Fique (2005) vincula la ausencia de procesos sociales incluyentes en la toma de decisiones, específicamente en el diseño de la política pública; Zicardi (2015) destaca las condiciones de habitabilidad que presentan las viviendas a nivel regional, destacando la percepción que tienen los habitantes de la calidad de vida que ofrece la vivienda de interés social.

Esta somera enunciación de trabajos en torno a la vivienda y a la habitabilidad sirve como objeto de estudio desvinculado a la sustentabilidad; existen pocos trabajos que vinculen la habitabilidad con la sustentabilidad y la vivienda de interés social; de ahí que este estudio busca abonarse a la discusión de esta línea de investigación abordando el caso de un conjunto urbano que formó parte de la política federal que pretendió fomentar la producción de vivienda sustentable.

\section{Diseño metodológico}

El análisis de este trabajo se llevó a cabo desde dos ejes metodológicos. El primero tomó información producida en documentos especializados sobre vivienda, vivienda sustentable y habitabilidad, a fin de definir conceptos, construir categorías de análisis, identificar variables e indicadores; al mismo tiempo, se revisaron los informes anuales publicados en la página electrónica de INFONAVIT en torno a los resultados arrojados por el programa Hipoteca Verde respecto a la vivienda sustentable.

En el segundo eje se hicieron uso de técnicas de campo como la observación, cuyo uso contribuyó a constatar la presencia de ecotecnologías visibles desde la parte exterior de la vivienda; éstas se incluirían en una lista de comprobación, la cual sería complementada con la información obtenida por la aplicación de entrevistas semi estructuradas a 35 residentes del conjunto urbano "Los Héroes San Pablo II" Tecamac, a fin de conocer sobre las ecotécnias que forman parte de la vivienda: focos ahorradores, WC ahorradores, válvulas de agua ahorradoras en las regaderas y fregaderos, uso de paneles solares; así como sobre la habitabilidad en torno a variables, como dimensión de la vivienda, número de recámaras, número de habitantes en la vivienda, número de ocupantes por recámara, existencia de privacidad entre los integrantes de la familia.

Con estas dos técnicas se comprobó la presencia de ecotécnias en dichas viviendas; también se constataron los indicadores de habitabilidad relacionados con la calidad de los materiales de construcción, dimensiones de la vivienda, hacinamiento y problemas sociales que se desprenden de estas condiciones de vida. 


\section{De la casa a la vivienda}

A lo largo del proceso evolutivo de la humanidad, ésta ha intervenido el territorio no sólo generando transformaciones físicas, adecuaciones y domesticaciones de los espacios naturales para ocuparlos, hacerlos habitables y organizados atribuyéndoles sentidos para su uso, sino también les asignó simbolismos, signos, significados y significantes que dieron forma a sistemas culturales y de comunicación que imprimieron dinamismo al todo social.

De acuerdo con Hegel (2001), en la casa se han conjugado las manifestaciones de la naturaleza orgánica, el contenido teológico-espiritual y hasta su representación a través de sus materiales de construcción, dimensiones y distribución de los espacios con base a cierta utilidad y funcionalidad de la vida cotidiana; asimismo, se establecieron nuevas formas de relación e interacción entre sus integrantes y su entorno, donde la traza de la ciudad se constituyó como una de las más claras manifestaciones de apropiación del espacio en diferentes momentos históricos: "es en la casa, con sus muros, pilares y vigas encadenados de modo cristalino y racional, donde se ven realizados estos rasgos esenciales en sus relaciones, sean estas simples, graves grandiosas o agraciadas. La naturaleza misma de estas relaciones no se dejan reducir a determinaciones numéricas y medidas precisas" (Hegel, 2001: 85).

La casa es una expresión material cargada de simbolismos en donde todo ser humano busca llevar a cabo sus sueños, como bien comenta Bachelard (2000). Todo espacio realmente habitado lleva como esencia la noción de casa. Ésta pasó a ser una extensión del ser humano, en donde se experimenta una disposición emocional, no sólo por sentir protección, sino también privacidad, pues se considera un espacio donde se puede descansar y aislarse del ajetreo del exterior; permite la convivencia y el fortalecimiento de lazos con los demás integrantes; además, se manifiestan diversos sentimientos y emociones, como la tristeza, la pena, la alegría, el orgullo de formar parte de un estatus social.

La noción y el significado social que la casa presentó hasta antes del desarrollo del capitalismo en su construcción física, la distribución de los espacios, dimensiones, funciones, significado y significante están completamente cambiados; de acuerdo con Engels (1873: 326), la transformación acelerada del modo de producción feudal al capitalista conllevó innovaciones de la base económica, sistemas productivos, estructura urbana y cambios sociales en el consumo, cultura e identidad; sólo por citar algunos.

El cambio del modo de producción no sólo propició la transformación de la base productiva y de los procesos de producción, sino también buscó dar fin a la herencia feudal de los asentamientos humanos; es decir, se inició el ensanche de las ciudades medievales, que consistió en destruir sus murallas y reconstruirlas de manera horizontal y abiertas, lo cual dio pie a la aparición y desarrollo de la industria de la construcción; actividad productiva estrechamente relacionada con el sistema capitalista en las principales ciudades europeas. "Por intermedio de Haussmann, el bonapartismo explotó extremadamente esta 
tendencia en París, para la estafa y el enriquecimiento privado. Pero el espíritu de Haussmann se paseó también por Londres, Manchester y Liverpool; en Berlín y Viena parece haberse instalado como en su propia casa" (Engels, 1873: 327).

\section{Vivienda}

En este contexto, la vivienda fue constituida como una mercancía inherente al proceso evolutivo del hombre y las ciudades que respondió a las fuerzas del mercado. Esta mercancía desarrolló uno de los negocios más redituables en las sociedades modernas y, “al convertirse en un negocio, la construcción iría poco a poco introduciendo todos los principios desarrollados en la producción industrial con el objetivo de minimizar costes y maximizar beneficios: economía de escala, no se construye una casa sino barrios enteros" (Ullán, 2014: 69). El significado que la casa tiene con relación a la vivienda dista mucho:

\footnotetext{
No es lo mismo la vivienda a la casa, existe una gran diferencia; la vivienda se ha tomado como definición del hecho edificado y construido en un lenguaje puramente arquitectónico, y la casa como lugar de la existencia, de la construcción del sujeto, del sentir propio de la experiencia humana. Por lo tanto, existe en la casa un territorio del cuidado de sí, de lo propio, el cual consolida lo que algunos teóricos de las ciencias sociales han llamado, desde la antropología, la configuración de una geografía humana que se posa sobre la superficie de la tierra y genera la intimidad del sujeto y la organización social del territorio (Sañudo, 2013: 216).
}

El sentido de mercancía es uno de los principios que va a diferenciar a la casa de la vivienda; el desarrollo del capitalismo convirtió a la casa en una mercancía altamente demandada durante la Revolución Industrial al incrementar los flujos migratorios hacia las ciudades que presentaron un sistema industrial con alta demanda de fuerza de trabajo. Conforme se complejizó el crecimiento urbano de las ciudades, se demostró que

\footnotetext{
el simple mercado no había conseguido construir ciudades armónicas y parecía evidente que por sí solo nunca lo haría. El Estado se plantea entonces intervenir en dos dimensiones de lo urbano: a) la planificación del crecimiento de la ciudad en sí, regulando el uso de los terrenos para funciones determinadas y b) la incentivación de vivienda barata con unos estándares de calidad suficientes para las clases trabajadoras. De la primera necesidad surgirán los instrumentos de la zonificación y de los planes de urbanismo. De la segunda, los programas (directos o indirectos) de vivienda protegida. Para poder intervenir y regular ambas dimensiones los estados irían progresivamente creando un complejo aparato burocrático y una creciente legión de técnicos especializados en los diferentes aspectos que una empresa de ingeniería social como aquella implicaba (arquitectos, ingenieros, abogados, economistas, topógrafos, geógrafos) (Ullan, 2014: 89).
}

\section{Vivienda de interés social}

A partir de la participación del Estado, la vivienda fue considerada un elemento igual que el entorno, es decir, 
aluden a un conjunto de alteraciones resultantes de la economía: la distribución del capital, las formas de poder, la administración de lo público y los aspectos culturales de la sociedad, que determinan la relación espacial entre lo íntimo y lo público. Es por esto que el conjunto de transformaciones y disfunciones del medio biofísico van de la mano con la forma de vida social; en este sentido, los seres humanos, en tanto seres naturales y culturales, son los receptores últimos de los impactos y las alteraciones ambientales (Sañudo, 2013: 219).

Con la participación del Estado en la producción de vivienda, se constituyó otra forma de tipificarla; en el caso de México, se comenzó a denominar como "vivienda de interés social"; de acuerdo con Puebla (2002) y Abiko (1995), surge como una iniciativa del Estado que va dirigida a resolver el problema de la vivienda de los sectores populares a través de distintos organismos gubernamentales. Generalmente, son proyectos que han sido materializados a través de la figura legal denominada "conjuntos habitacionales", los cuales fueron construidos de manera horizontal y vertical.

La Ley Federal de Vivienda, en su artículo $3^{\circ}$, establece: "para todos los efectos legales, se entiende por vivienda de interés social aquella cuyo valor, al término de su edificación, no exceda de la suma que resulte de multiplicar por diez el salario mínimo general elevado al año, vigente en la zona que se trate" (Diario Oficial de la Federación, 1984). De esta definición destaca la importancia que se le da al número de salarios de los posibles beneficiarios. De este modo, se llevó a cabo una tipología de la vivienda que tuvo como base cierto número de salarios mínimos, tal como se aprecia en la Alianza para la Vivienda 19952000, pues manejó un incremento a 15 salarios mínimos y propuso la vivienda popular con 25 salarios mínimos.

Para el 2006, la Comisión Nacional de Fomento a la Vivienda (CONAFOVI) destacó a la vivienda como un elemento fundamental de la calidad de vida, la accesibilidad, el entorno ambiental y el carácter único de una comunidad, contribuyendo a dar sentido al lugar. La forma en que las casas son diseñadas y construidas, el conjunto planeado y edificado y las áreas verdes y espacios abiertos localizados y conservados son factores que determinan si una comunidad es sustentable ambientalmente (CONAFOVI, 2006).

Por consiguiente, el gobierno federal, a través de INFONAVIT, fungió como el principal productor de vivienda y urbanizador en el país hasta 1992; año en el que la reforma cedió a la iniciativa privada dicho papel y se limitó a su financiamiento; posteriormente, en 2008, se puso en marcha el programa Hipoteca Verde con el fin de promover la construcción de vivienda sustentable, y no sólo cumplió con los lineamientos propuestos en el ámbito internacional en torno a impulsar medidas de mitigación que contribuyeran a reducir las afectaciones al medio ambiente, sino también sentó las bases para impulsar el desarrollo sustentable de los recursos naturales aplicados a la vivienda. 


\section{Vivienda sustentable}

La noción de vivienda sustentable es producto de diversas reuniones de actores internacionales preocupados por proteger y preservar el medio ambiente, así como por impulsar el desarrollo de una cultura que promueva el uso racional de los recursos naturales y la reducción de la contaminación del suelo, del aire, del agua, entre otros.

“Bajo la dirección de la Sra. Brundtland se da a conocer, en 1987, el informe de la Comisión Mundial para el Medio Ambiente y Desarrollo (WCED, 1987), un grupo independiente pero creado a solicitud de la Asamblea General de las Naciones Unidas para elaborar una "agenda global para el cambio". En dicho informe, Our Common Future, se examinan los problemas más críticos en torno al desarrollo y el medio ambiente y se indican propuestas de solución. A partir de allí se difunde el término de desarrollo sustentable como "aquel que responde a las necesidades del presente de forma igualitaria, pero sin comprometer las posibilidades de sobrevivencia y prosperidad de las generaciones futuras", y se establece que la pobreza, la igualdad y la degradación ambiental no pueden ser analizados de manera aislada" (Foladori y Tommasino, 2000: 44).

Desde este informe se comenzó a incorporar el concepto de sustentabilidad en el ámbito económico, principalmente a los sistemas productivos de los distintos sectores, sub sectores y ramas productivas, con el fin de reducir sus efectos al medio ambiente; lo mismo sucedió en la prestación de servicios turísticos y recreativos, entre otros, y, por supuesto, también se incorporó a la producción de vivienda.

Este informe sería punto de referencia en el desarrollo de diversas reuniones internacionales en donde se discutieron problemas vinculados al deterioro del medio ambiente, como: en Río de Janeiro en 1992, el Protocolo de Kioto sobre cambio climático y gases de efecto invernadero en 1997, la Cumbre del Milenio de las Naciones Unidas en el 2000, la Cumbre Mundial sobre el Desarrollo Sostenible en 2002, la conferencia de la ONU sobre el cambio climático en 2010 y la conferencia Habitat III en 2016; de esta última se desprendió la Nueva Agenda Urbana donde se colocó a la vivienda como elemento central para alcanzar el desarrollo urbano sostenible. En las últimas décadas, se ha vinculado a la vivienda con las dimensiones de la sustentabilidad.

\footnotetext{
El componente social de la sustentabilidad en la vivienda está relacionado con la calidad de vida, pues la vivienda no es solamente un espacio físico: es, sobre todo, el espacio donde las personas desarrollan una parte fundamental de su vida; donde se efectúan las actividades más fundamentales (comer, dormir, higiene); donde se construye gran parte de su individualidad y de sus relaciones privadas. Por ello, la calidad de la vivienda y de su entorno es crucial para el buen desenvolvimiento de las personas en lo personal y en la comunidad (Fundación IDEA, 2013: 42).
}

El diseño climático, el espacio habitable, la distribución y el sentido de los espacios de la vivienda sustentable se han asociado al grado de satisfacción, así como a la privacidad 
de sus residentes; y a su vez, todo ello se relaciona con las condiciones que presentan el entorno, la movilidad, la accesibilidad, los servicios públicos, los espacios educativos, la salud, las fuentes de empleo, la recreación y el fortalecimiento de los vínculos comunitarios.

El componente económico de la sustentabilidad está relacionado con los costos y beneficios económicos asociados a la vivienda, sus servicios y su ubicación. En primer lugar, es importante destacar que la adquisición o renta de una vivienda es una de las decisiones económicas más relevantes en la vida de las personas, dados los costos directos e indirectos de esta decisión. La propiedad de una vivienda constituye un factor fundamental de la estabilidad económica de las familias y uno de los principales amortiguadores frente a momentos de estrés económico -cuando la compra no representa un costo financiero que supera la capacidad de las familias-. La plusvalía de la vivienda en el mediano y largo plazo puede convertirse también en uno de los principales activos económicos de las personas (Fundación IDEA, 2013: 42).

El componente ambiental de la sustentabilidad se ha entendido como el medio con el que se intenta conformar una nueva cultura ambiental en la sociedad y, con ello, cambiar la manera en que se relacionan las personas con el entorno natural. De ahí que se haya tomado a la vivienda como el elemento que permitiría alcanzar dicho propósito. En México, los esfuerzos realizados para promover la producción de vivienda de interés social sustentable se enfocaron más a la dimensión económica, ya que, aunque se ha buscado incorporar la dimensión social y el medio ambiental, se encuentran desarrolladas de manera incipiente.

Las dimensiones de la sustentabilidad no sólo deben estar integradas en la vivienda, sino también en su entorno; si no se pone atención en ello, serían acciones aisladas e insustentables. De conseguirlo, no sólo se beneficia de manera significativa el consumo de recursos naturales y energéticos, que en lo general reduciría el impacto al medio ambiente, sino también la habitabilidad de la vivienda misma.

\section{Vivienda sustentable como concepto}

Referirse a la vivienda sustentable como concepto es adentrarse a una diversidad de percepciones que se tiene de ésta, dependiendo del enfoque disciplinario desde donde se esté definiendo. De acuerdo con Sola et al. (2005), las viviendas sostenibles son saludables por no tener ningún material o tecnología que pueda alterar nuestro bienestar, salud o estado de ánimo; son ecológicas porque todos los materiales y las tecnologías empleados en su construcción son ecológicos; son de alta eficiencia energética, porque consumen tan sólo el 20\% de lo que lo hace una vivienda similar; son bioclimáticas y, además, son económicas por costar lo mismo que cualquier otra casa de calidad de su misma especie.

“De acuerdo con la OCDE (2003), las viviendas sustentables son aquellas edificaciones que tiene un mínimo impacto en el medio ambiente. Además de estar diseñada para tener bajo impacto ambiental, ser funcional, estética, perdurable y adaptable, satisfacer eficientemente las necesidades de sus residentes" (Valdés, 2009: 315). 
Para el caso de México, se entendió y se vinculó a la vivienda sustentable con los principios de las Acciones de Mitigación Nacionalmente Apropiadas (NAMAS, por sus siglas en inglés); así, se planteó el desarrollo de acciones "desde el interior de las viviendas, logrando que éstas tengan un consumo eficiente de recursos. Por ello, se han creado algunos mecanismos que ayudan a la población a incorporar el uso de ecotecnologías al interior de sus viviendas, tales como la Hipoteca Verde, dirigidas hacia la vivienda y el Programa 'Ecocasa'"' (Secretaría de Gobernación, 2014).

Con "la NAMA se pretendió mitigar las emisiones en el sector de la vivienda al proveer financiamiento adicional para mejorar la eficiencia energética y disminuir el consumo de combustibles fósiles y del agua. Lo cual se logra a través de la implementación de eco-tecnologías, mejoras en el diseño arquitectónico y la utilización de materiales constructivos eficientes" (CONAVI, SEMARNAT, 2012: 1); ello, traería como consecuencia no sólo el beneficio al medio ambiente, sino también un incremento en la calidad de vida.

Para efectos de este trabajo, se adopta la concepción que las NAMAS han instituido como vivienda sustentable, pues fueron tomadas como fundamento para que el gobierno federal pusiera en marcha programas de fomento a la vivienda sustentable, principalmente Hipoteca Verde y Ecocasa. "Los dos programas otorgan financiamiento adicional o subsidio para cubrir el costo incremental de distintas medidas encaminadas a la reducción de consumo de energía eléctrica, gas y agua, como el aislamiento, calentador solar de agua y equipos, aparatos o accesorios de bajo consumo de energía y agua en viviendas nuevas" (CONAVI, SEMARNAT, 2012: 2).

Con el fomento de la producción de viviendas sustentables, se pretendió generar un cambio cultural en sus residentes al promover una relación más amigable con el entorno natural, especialmente con los recursos no renovables que más consume el ser humano; por ejemplo, buscar el uso eficiente y racional del agua, reducir el impacto en la contaminación del aire al sustituir calentadores de agua que usan gas LP por calentadores solares y disminuir el consumo de energía eléctrica al sustituir las lámparas normales por focos ahorradores. Todo ello vinculándolo con el diseño de la vivienda, así como con los niveles de satisfacción, tanto al interior como por el equipamiento urbano disponible en su entorno.

\section{Habitabilidad}

El tema de habitabilidad se vuelve complejo si se toma en cuenta la diversidad de disciplinas que la han tomado como objeto de estudio, ya que cada una sólo destaca características de su interés. De ahí que nos limitaremos a describir algunas nociones de la habitabilidad en la vivienda, la cual forma parte de la habitabilidad urbana. 
En la Edad Media, la habitabilidad estuvo asociada con problemas de salud pública que se manifestaron en Europa. "Hasta entrado el siglo XIX, las ciudades europeas presentaban un sin número de enfermedades como hepatitis, cólera, tuberculosis, etcétera, debido al descontrol de las aguas residuales, a los espacios habitacionales poco ventilados, a la suciedad y al hacinamiento de las personas más pobres [...] problema que a raíz de la Revolución Industrial se agudizó" (Moreno, 2008: 48). En este escenario, el actor que asumiría el papel protagónico en la transformación de esta realidad fue el Estado, pues llevó a cabo transformaciones en sus funciones y comenzó a diseñar una política pública dirigida a consolidar la estructura urbana de las ciudades a fin de reducir los impactos de estas enfermedades. La vivienda fue uno de los objetivos en el diseño de estrategias para mejorar su habitabilidad.

En la Declaración Universal de los Derechos Humanos de 1948, en su artículo 25, se establece el derecho que tiene toda persona a tener calidad de vida, asociado al acceso a la salud, bienestar, alimentación, vivienda, servicios, entre otros. De acuerdo con la Organización de las Naciones Unidas, la habitabilidad guarda relación con las características y cualidades del espacio, entorno social y medio ambiente que contribuyen singularmente a dar a la gente una sensación de bienestar personal y colectivo, e infundirle la satisfacción de residir en un asentamiento determinado (Molar y Aguilar, 2013). Esta concepción de la habitabilidad se vincula a la habitabilidad urbana, pues se incluyen las condiciones del entorno como elementos que contribuyen a mejorar la calidad de vida de los habitantes de las ciudades, barrios, colonias, entre otras.

Con relación a la habitabilidad en la vivienda, "la oficina del Alto Comisionado para los Derechos Humanos define a la habitabilidad de la vivienda a partir de sus características físicas y señala que la 'vivienda no es adecuada si no garantiza seguridad física o no proporciona espacio suficiente, así como protección contra el frío, la humedad, el calor, la lluvia, el viento, u otros riesgos para la salud y peligros estructurales'" (ACNUDH, 2010: 4; Zicardi, 2015: 37). Esta noción de habitabilidad de la vivienda pone énfasis en los atributos físicos y adolece de elementos subjetivos, como la privacidad de los habitantes, el sentido, la dimensión y la distribución de los espacios, el confort, la estabilidad emocional y psicológica, entre otros.

Otros autores, como Mercado (2004), incluyen en sus propuestas la parte subjetiva que no se había considerado:

La habitabilidad es el grado en que la vivienda se ajusta a las necesidades y expectativas de sus moradores.

La relación entre la habitabilidad y grado de control sobre el entorno, los niveles de activación, la tasa de información y el valor hedónico del diseño, así como la relación entre los factores señalados y los físicos del diseño, usan al sujeto como instrumento de medición. Concluye que la habitabilidad de la vivienda está determinada por correlatos físicos y psicológicos, que interactúan e influyen entre sí (Mercado, 2004; citado en Molar y Aguirre, 2013: 74). 
Para el caso de este trabajo, nos limitaremos a entender la habitabilidad de la vivienda como los factores materiales e inmateriales en los que desarrollan su cotidianidad los residentes; entran en juego los materiales de construcción; las dimensiones; el hacinamiento; los servicios básicos, de información y de comunicación; la disponibilidad de muebles; el desarrollo de los habitantes; la privacidad; el confort; la percepción y el grado de satisfacción o placer que provoca a sus habitantes.

Estos mismos indicadores se emplean para medir la habitabilidad de la vivienda sustentable, pero se sumarían los vinculados al medioambiente; es decir, la disponibilidad de recursos naturales y energéticos que tienen relación directa con las ecotecnologías y su presencia en las viviendas; con ello, permitirán identificar el nivel de satisfacción que perciben sus habitantes, así como los efectos que producen al medio ambiente desde dos perspectivas: el consumo de recursos naturales y energéticos, y los contaminantes derivados de las viviendas aunque estén dotadas de ecotécnias.

\section{Programa Hipoteca Verde}

"Según la Organización de las Naciones Unidas, la habitabilidad guarda relación con las características y cualidades del espacio, entorno social y medio ambiente, que contribuyen singularmente a dar a la gente una sensación de bienestar personal y colectivo, e infundirle la satisfacción de residir en un asentamiento determinado" (Molar y Aguirre, 2013: 74). Bajo estos lineamientos, en México, el gobierno federal incorporó en la vivienda sustentable algunos de estos principios.

El Programa Nacional de Vivienda 2007-2012 del Gobierno del Presidente Felipe Calderón (2006-2012), inspirado en los principios del Desarrollo Sustentable se planteó, entre otros objetivos, "impulsar un desarrollo habitacional sustentable" (CONAVI, 2008: 37). Con esta base, a partir de 2007, el Instituto del Fondo Nacional de la Vivienda para los Trabajadores (en adelante INFONAVIT) impulsó el programa de financiamiento Hipoteca Verde, definido como:

Un crédito que cuenta con un monto adicional para que el derechohabiente pueda adquirir una vivienda con tecnologías eficientes que disminuyen el consumo de agua, energía eléctrica y gas, o incorporarlas a la misma en caso de que la vivienda a adquirir no cuente con ellas en el momento de la formalización del crédito, con el objetivo de mejorar la calidad de vida de nuestros acreditados, disminuyendo su gasto familiar y las emisiones de CO2 al medio ambiente (INFONAVIT, 2011: 5; López et al., 2015: 47).

Este programa se ha desarrollado en tres fases: como proyecto piloto, desde la formalización del primer crédito (julio de 2007) hasta el 4 de marzo de 2008; de esta fecha hasta el 31 de diciembre de 2010 (2010Q4), fue un programa opcional en el que podían participar todas las desarrolladoras inmobiliarias interesadas; y, finalmente, desde enero de 2011 es un programa obligatorio para todos aquellos que deseen obtener un crédito del INFONAVIT (INFONAVIT, 2011; López et al., 2015: 47). 
Los buenos resultados obtenidos de esta prueba piloto sentaron las bases para que en 2009 se institucionalizara y se pusiera en práctica en todo el país. A partir de este año, el INFONAVIT pretendió que el programa operara como un esquema de crédito opcional para financiar la adquisición de un paquete fijo de ecotecnologías dependiendo de la zona climática. Desde 2011 se puso en marcha el programa Hipoteca Verde Flexible, el cual permitió a los derechohabientes seleccionar las ecotecnologías que satisficieran mejor sus necesidades.

De acuerdo con INFONAVIT (2011) para que la institución otorgue un crédito a un derechohabiente, es indispensable la instalación de tecnologías sustentables en la vivienda. La opción para financiar estas ecotecnologías es mediante un crédito adicional de Hipoteca Verde cuyo importe está en función del ahorro mínimo progresivo y del nivel de ingresos del trabajador. La deuda final de los usuarios equivaldrá a la suma de ambos créditos: de vivienda y de HV9 (López et al., 2015: 51-52).

Para definir a la vivienda como sustentable, INFONAVIT (2010) toma en cuenta ocho principios: compacta (de tipo vertical), con acceso a Internet, ecológica (por ser de Hipoteca Verde, con diseño bioclimático en materiales y procedimientos), habitable (tamaño, flexible y para personas con discapacidad), equipada, incluyente, diversa y segura. Al mismo tiempo, toma en cuenta veinte características distribuidas en tres atributos: entorno próspero, vivienda de calidad y comunidad solidaria y responsable.

Con el paso del tiempo, INFONAVI, además de vincular a la vivienda sustentable a las ecotecnologías promovidas por los programas de ecocrédito (Hipoteca Verde y Ésta es tu Casa), también la ha relacionado con otros planes, como el de Competitividad Municipal, con el cual se pretende identificar el Grado de Sustentabilidad de los municipios a través del fortalecimiento y la promoción de las mejores prácticas en materia de habitabilidad, competitividad, gestión y buen gobierno, y solvencia financiera.

La oferta de vivienda sustentable que los distintos programas federales han pretendido promover se constituye por programas de ecocréditos (equipamiento de la vivienda por ecotecnologías y dispositivos ahorradores) y de Competitividad Municipal, los cuales forman parte de los lineamientos, de las políticas nacionales, estatales y municipales que constituyen la política urbana y tienen como propósito impulsar la sustentabilidad de la vivienda.

Esta política urbana se fundamenta legalmente en la Constitución Política de los Estados Unidos Mexicanos, la Ley General del Equilibrio Ecológico, la Ley de Vivienda, la Ley de Planeación, la Ley General de Asentamientos Humanos, el Código de Vivienda, el Plan Nacional de Desarrollo 2007-2012, el Programa Nacional de Vivienda 2007-2012, el Programa Específico para el Desarrollo Habitacional Sustentable ante el Cambio Climático, los programas sectoriales, la Estrategia nacional de cambio climático, entre otras más. 
El programa de Hipoteca Verde es entendido como "el crédito hipotecario INFONAVIT con un monto adicional para que el derechohabiente pueda adquirir una vivienda con tecnologías para el uso eficiente de agua, a través de la incorporación de llaves ahorradoras, regaderas ahorradoras, WC ahorradores, etc., gas LP y energía eléctrica" (INFONAVIT, 2016); por ello, en este trabajo nos limitamos a estas tres variables.

\section{Caso los Héroes San Pablo II Tecamac, Estado de México}

El conjunto urbano Los Héroes San Pablo II se ubica en calle sin nombre y sin número en el Ejido de San Pablo Tecalco Municipio de Tecámac, Estado de México. El grupo SADASI llevó a cabo su desarrollo para alojar 1,549 viviendas, conforme a los Planos de Lotificación, en una superficie de 172,839.08 m2 (GEM, 2016). En 2016, “Los Héroes San Pablo” se certificó como con un conjunto habitacional sustentable.

Forman parte integrante de la presente autorización para todos los efectos legales y de acuerdo a [sic] las siguientes características generales: superficie habitacional vendible: $128,433.37 \mathrm{~m}^{2}$ superficie de comercio de productos y servicios básicos vendible: $898.98 \mathrm{~m}^{2}$ superficie de donación municipal: 19,091.61 m² (incluye $503.61 \mathrm{~m} 2$ de donación adicional). Superficie de restricción de construcción por talud: 6,335.16 m² superficie de vías públicas: 18,079.96 $\mathrm{m}^{2}$, superficie total del conjunto urbano: 172,839.08 $\mathrm{m}^{2}$ superficie fuera de autorización: 175,244.15 $\mathrm{m}^{2}$, superficie total de terreno: 348,083.23 $\mathrm{m}^{2}$ número de manzanas: 8 número de lotes: 69 número de viviendas: 1,549 (GEM, 2016).

Las viviendas construidas oscilan entre los 60 y los 80 metros cuadrados; se encuentran integradas por sala, comedor, cocina, un baño completo, un medio baño, dos recámaras, un cuarto de servicio y un cajón de estacionamiento; cuentan con planta baja y un piso, y están diseñadas para familias no mayores a cuatro integrantes.

De acuerdo con información presentada en su página de Internet, el grupo SADASI (s/f) fue pionero en la aplicación del programa Hipoteca Verde del INFONAVIT, el cual se diseñó con el fin de promover la instalación de tecnologías para el ahorro de agua y energía en las viviendas. Según SADADSI, uno de los mayores compromisos del Grupo es el respeto por la ecología y el medio ambiente. Para ello, han dedicado tiempo y recursos importantes a la investigación e implementación de medidas para crear desarrollos urbanos integrales y sustentables que contribuyan a la conservación del medio ambiente.

\section{Discusión de resultados}

El análisis de la sustentabilidad y habitabilidad de la vivienda en este conjunto urbano se desarrolló en dos momentos. En el primero se tomó como elemento de estudio los informes que INFONAVIT publicó en su sitio web. Los informes de Hipoteca Verde que promueven la producción de vivienda sustentable se basan en el cálculo de los ahorros 
económicos y la disminución en el consumo de agua, gas y electricidad, así como en el equipamiento e infraestructura destacando el uso de ecotécnias.

Un primer resultado que se pudo desprender tiene que ver con la metodología empleada por INFONAVIT, al tomar en cuenta para su medición sólo algunos puntos de lo energético y al recurso agua, sin considerar suelo, aire, flora, fauna, residuos, autosuficiencia, estética, integración social, impacto ambiental desde su creación hasta su remodelación y deshecho (ciclo de vida de la vivienda), diseño, impacto humano en la salud, economía, confort ni otras características primordiales y básicas en el marco metodológico para la evaluación de la sustentabilidad.

El desarrollo del segundo momento se relaciona con el trabajo de campo realizado, en el cual se aplicaron entrevistas a residentes de la vivienda sustentable a fin de identificar la calidad de vida que ofrece desde sus condiciones de habitabilidad; asimismo, se usó la técnica de la observación y una lista de cotejo; los resultados se agrupan en las variables, como materiales de construcción, presencia de ecotecnologías en la vivienda, hacinamiento, privacidad y confort.

Con la revisión de los informes existentes en el portal de INFONAVIT, se pudo identificar que hay ausencia de éstos en algunos años, desde su origen hasta la actualidad; también se observó en la metodología empleada por INFONAVIT para la medición de la sustentabilidad que carecen de una continuidad de sus variables en cada uno de ellos, cuya situación complica la identificación de criterios, indicadores e índices que permitan dar seguimiento de las variables en cada reporte publicado.

La medición de la sustentabilidad de la vivienda se centra en tres variables: recurso agua, gas LP y energía eléctrica, las cuales están vinculadas al ámbito económico, es decir, se destaca el ahorro monetario de los habitantes de las viviendas sustentables por el uso de ecotecnologías y no se incluyen aspectos sociales, culturales y ambientales de manera desarrollada.

El mayor logro de las viviendas sustentables se vincula al ámbito económico, debido a la reducción de costos en lo energético por el uso de ecotecnologías, tal es el caso del gas LP y de la energía eléctrica; asimismo, se ha reducido hasta un 50\% el pago de energía eléctrica por la sustitución de focos incandescentes por ahorradores, y en la tarifa del agua, pues se presenta un ahorro significativo por el uso de WC ahorradores, llaves de agua ahorradoras, regaderas ahorradoras, entre otras ecotécnias; sin embargo, el impacto económico más significativo se aprecia en el uso de calentadores solares, ya que, en caso de tener tanque estacionario, representa dejar de pagar recargas mensuales de gas LP y sólo realizarlas cada seis $\mathrm{u}$ ocho meses $\mathrm{y}$, si se tiene cilindro de veinte litros, éste se llega a extender hasta tres meses.

Parece contradictoria esta lógica económica de la vivienda, es decir, por un lado, se consigue reducir el gasto económico por usar ecotécnias, pero, por otro, su construcción se 
hace en suelo lejano de los espacios laborales; de equipamientos comerciales; de servicios: recreativos, educativos, deportivos, entre otros; por consiguiente, se incrementan los costos de traslado relacionados con el consumo de combustibles, cuyo hecho constituye situaciones de insustentabilidad por el alto consumo de hidrocarburos asociado al número de viajes al día que deben realizar los residentes a cada lugar.

Derivado del trabajo de campo, se identificaron las condiciones de habitabilidad que presentan las viviendas de este conjunto urbano; la constante de los entrevistados es la mala calidad de los materiales con que están construidas; destacan las filtraciones de agua en techos, ya que es un problema que enfrentan cada temporada de lluvias; al mismo tiempo, enfatizan la velocidad de su deterioro, pues, aun sin terminar de pagarlas, su reparación costaría lo mismo que si construyeran otra nueva.

Del total de viviendas entrevistadas, el $68 \%$ presenta problemas de hacinamiento. Están diseñadas para cuatro habitantes, pero se llega a identificar un número mayor. Sin embargo, tanto el productor de la vivienda como INFONAVIT pueden argumentar que el problema no es de ellos, ya que las dimensiones son adecuadas para las personas calculadas. Fue posible constatar que las viviendas que presentan esta situación son las familias grandes y aceptan estas condiciones por no contar con recursos económicos que les permitan adquirir una vivienda adecuada al número de integrantes.

Derivado del hacinamiento, se presentan otros problemas, por ejemplo: adaptar el cubo de la escalera como una recámara más o transformar por las noches las salas como dormitorios; este escenario deriva en la falta de privacidad; situación que no sólo deteriora la calidad de vida de quien habita estos espacios, sino también las relaciones familiares.

Asimismo, producto del hacinamiento, los integrantes más jóvenes toman las calles o espacios públicos como puntos de reunión, que, en la mayoría de los casos, inducen al alcoholismo, drogadicción, pandillerismo e, incluso, los ha llevado a delinquir en diferentes niveles de complejidad.

Otro problema referente al hacinamiento lo representan las mascotas, ya que la mayoría de las viviendas cuentan con gatos o perros con los que comparten el espacio; en algunos casos, tienen hasta tres mascotas; este hecho puede derivar en problemas de salud para la familia, pues no tienen los hábitos de limpieza para evitar malos olores, pelaje en el interior y exterior de las viviendas o heces fecales.

Cabe mencionar que lo que en nuestro país se entiende como vivienda sustentable se limita a la implementación de ecotecnologías, pero se encuentra muy alejada de los cánones internacionales y más aún de cumplir con la habitabilidad de la vivienda; no se debe perder de vista que está diseñada para familias pequeñas y, en la mayoría de los casos, rebasa por mucho el número de residentes para el que fueron diseñadas; se encontraron casos en donde se encontraba más de una familia en una vivienda. 


\section{Conclusiones}

La vivienda sustentable promovida por INFONAVIT con el uso de ecotecnologías cumple de manera parcial con lo que se establece como una vivienda sustentable, ya que se limita a la dotación de ecotecnologías que impacten en el ahorro de energía eléctrica, el consumo de gas LP y del recurso agua.

La vivienda sustentable tiene buena aceptación por los habitantes por reducir los costos en consumo de gas, energía eléctrica y agua potable; sin embargo, la mayor parte de los entrevistados coinciden en que están en esos espacios porque no les queda otra alternativa y, si pudieran, cambiarían sus viviendas por otras más grandes y con mejores condiciones físicas.

La vivienda sustentable de interés social, fomentada por INFONAVIT, favorece al medio ambiente tanto en la reducción del consumo de agua como de emisiones de bióxido de carbono, lo cual contribuye a aminorar el efecto invernadero que cada vez se hace más problemático en el planeta.

Este tipo de vivienda debe mejorarse en cuanto a lo físico; es decir, utilizar en sus construcciones materiales de mejor calidad que no sólo se limite a la sustentabilidad, sino abrirse al entorno al ampliar las áreas verdes, construir los conjuntos urbanos cerca de los equipamientos, respetar los usos de suelo y dar mantenimiento a las áreas comunes, como jardines, espacios deportivos, andadores y calles, entre otros.

Es importante incorporar en la vivienda sustentable elementos sociales que fomenten las relaciones sociales cordiales intrafamiliares y transfamiliares; los espacios del entorno deben ser amigables y funcionales con sus residentes, pues muchos de los entrevistados comentan que los espacios comunes han sido tomados por jóvenes que se alcoholizan e, incluso, se drogan haciendo de estos lugares zonas de riesgo.

Al no planificar la vivienda sustentable e incorporarla en proyectos urbanos más amplios, es evidente que no cuenta con ningún proyecto de separación de aguas grises y residuales, ni con planes para el manejo de residuos sólidos que ayuden a reducir el impacto ambiental.

Por tanto, la habitabilidad en este tipo de vivienda no es la adecuada. La vivienda presenta daños físicos, grietas, filtraciones, entre otros, así como graves problemas de convivencia por sus dimensiones, pues oscila entre los $44 \mathrm{~m} 2$ y los $90 \mathrm{~m} 2$. 


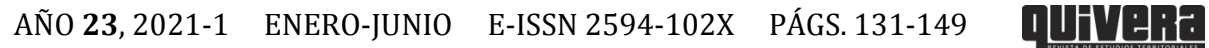

\section{Referencias}

Abiko, A. K. (1995). Introdução à Gestão Habitacional. Escola Politécnica da USP, Departamento de Engenharia de Construção Civil. Sao Paulo: USP. Recuperado de: http://www.pcc.poli.usp.br/files/text/publications/TT_00012.pdf

ACNUDH (2010). El derecho a una vivienda adecuada. Folleto informativo, 21. Geneva: Alto Comisionado de las Naciones Unidas para los Derechos Humanos. Recuperado de: https://www.ohchr.org/Documents/Publications/FS21_rev_1_Housing_sp.pdf

Bachelard, G. (2000), La poética del espacio, Buenos Aires: Fondo de Cultura Económica.

Cámara de Diputados del H. Congreso de la Unión, CESOP (2006). La vivienda en México. Construyendo análisis y propuestas, México: Cámara de diputados LIX Legislatura, Centro de Estudios sociales y de opinión pública

Coulomb, R. y Schteingart, M. (coords.) (2006), Entre el Estado y el mercado. La vivienda en el México de hoy, México, UAM, Miguel Ángel Porrúa.

CONAFOVI, (2006) Guía para el uso eficiente de la energía en la vivienda. Recuperado de: http://www.conalep.edu.mx/academicos/Documents/eficiencia_energetica/material/conafoviguia-ee-en-la-vivienda-2006.pdf

CONAVI, SEMARNAT (2012). NAMA Apoyada para la Vivienda Sustentable en México - Acciones de Mitigación y Paquetes Financieros. Recuperado de: http://www.conavi.gob.mx/images/documentos/sustentabilidad/NAMA_mexicana_de_vivienda_ sustentable_documento_espa\%C3\%B1ol.docx

De Mattos, C. (2015). Revolución urbana. Estado, mercado y capital en América Latina. Santiago, Chile: Ril Editores, Colección Estudios Urbanos UC.

Diario Oficial de la Federación (1984). Ley Federal de Vivienda. Recuperado de: http:/ /leyco.org/mex/fed/121.html\#c1

Eibenschutz, R. y Goya, C. (2009), Estudio de la integración urbana y social en la expansión reciente de las ciudades en México, 1996-2006. México: Sedesol, UAM, Miguel Ángel Porrúa.

Engels, F. (1873). Contribución al problema de la vivienda, t. 3 . Recuperado de: http://www.marxists.org/espanol/m-e/1870s/vivienda/

Fique Pinto, L. (2005). La habitabilidad de la vivienda de interés social en Colombia. Un enfoque en los procesos y las decisiones. Revista INVI, 20(55). Recuperado de: http://revistainvi.uchile.cl/index.php/INVI/article/view/317/883

Foladori G. y Tommasino, H. (2000). El concepto de desarrollo sustentable treinta años después, Desenvolvimento e Medio Ambente, 1(), jan/jun, Editora da UFPR.

Fundación Idea, (2013). Estrategia Nacional para la Vivienda Sustentable. Componente Ambiental de la Sustentabilidad, México. Recuperado de: http://conuee.gob.mx/pdfsvivienda/FIDEAEmbbritanicaestrategianacionalviviendasustentablef.pdf

GEM (2016). Gaceta de Gobierno. Registro DGC NÚM 0011021. Recuperado de: http://legislacion.edomex.gob.mx/sites/legislacion.edomex.gob.mx/files/files/pdf/gct/2006/dic26 2.pdf.

Hegel, G.W. (2001), La arquitectura, Barcelona, Kairós

INFONAVIT (2016). Manual Explicativo de la Vivienda Ecológica Hipoteca Verde. México: INFONAVIT. Recuperado de: http://portal.infonavit.org.mx/wps/PA_CsultaLicitaciones/MostrarArchivoBD?archivoBD=2858

INFONAVIT. (2011). Manual explicativo vivienda ecológica. Hipoteca Verde. México: INFONAVIT.

INFONAVIT. (2010). Informe de sustentabilidad. Recuperado de: http://portal.INFONAVIT.org.mx/wps/wcm/connect/INFONAVIT/el+instituto/INFONAVIT_en _cifras/Informes_anuales_de_sustentabilidad.

Lentini, M. y Palero, D. (1997). El hacinamiento: la dimensión no visible del déficit habitacional, Revista invi, 31(12), 23-32. Recuperado de: http://www.revistainvi.uchile.cl/index.php/INVI/article/view/220/742 
López Jiménez, D., Castillo Girón, V. M., Machuca Martínez, M., y Ayala Ramírez, S. (2015). Ecotecnología en las viviendas mexicanas: El Programa Hipoteca Verde y sus implicaciones en la rentabilidad de las empresas inmobiliarias. Revista de Economía del Caribe, (16). Recuperado de: http://rcientificas.uninorte.edu.co/index.php/economia/article/view/7172

Maya P., E. (1999). El sector privado y la vivienda de interés social en la Zona Metropolitana de la ciudad de México, México: impresora Deseret.

Maycotte, E. y Sánchez, E. (2009). Ciudades dispersas, viviendas abandonadas: la Política de vivienda y su impacto territorial $y$ social en las ciudades mexicanas. Recuperado de: http://upcommons.upc.edu/revistes/bitstream/2099/11919/1/04_PROCEEDINGS_M4_09_002 5.pdf

Mena, E. (2011). Habitabilidad de la vivienda de interés social prioritaria en el marco de la cultura, Cuadernos de Vivienda y Urbanismo, 4(8). https://doi.org/10.11144/Javeriana.cvu4-8.hvis

Molar, M. y Aguirre, L. (2013) ¿Cómo es la habitabilidad en viviendas de interés social? Caso de estudio: fraccionamientos lomas del bosque y privadas las torres en Saltillo, Coahuila, RICSH Revista Iberoamericana de las Ciencias Sociales y Humanísticas, 2(4), Centro de Estudios e Investigaciones para el Centro Docente A.C. Recuperado de: https://www.redalyc.org/articulo.oa?id=503950746004

Montejano E. J. A., Caudillo C. C. A. y Cervantes S. M. (2018) Vivienda de interés social, segregación residencial y accesibilidad: análisis de 121 conjuntos urbanos en el arco norte del Valle de México, 2001-2010, Revista Estudios Urbanos, 33(1), 187-224. Recuperado de: http://www.scielo.org.mx/pdf/educm/v33n1/2448-6515-educm-33-01-00187.pdf

Moreno, S. (2008). La habitabilidad urbana como condición de calidad de vida, Palapa Revista de Investigación Científica en Arquitectura. 3(2). II, Universidad de Colima, México. Recuperado de: https://www.redalyc.org/pdf/948/94814774007.pdf

Narbondo, P. (2014.). Estado neoliberal, Estado desarrollista y Estado de bienestar universalista. Universidad de la República Montevideo, Uruguay. Recuperado de: https://www.colibri.udelar.edu.uy/jspui/bitstream/20.500.12008/4575/1/DOL\%20CP\%2002\%2014. pdf

Orozco, I. y Guzmán, S. (2015). Reflexiones sobre la habitabilidad de la vivienda social. El Área Metropolitana Centro Occidente, Colombia. Revista Bitácora Urbano Territorial, 25(1), 27-35. Recuperado de: https:/ / www.redalyc.org/articulo.oa?id=74841604003

Puebla, C. (2002). Del intervencionismo estatal a las estrategias facilitadoras. Cambios en la política de vivienda en México (1972-1994), México, El Colegio de México, A.C.

SADASI (s/f) Los Héroes. Recuperado de: https://www.losheroes.mx/HeroesR1/grupo-sadasi/desarrollossustentables-sadasi/

Sañudo, L. (2013). La casa como territorio. Una nueva epistemología sobre el hábitat humano y su lugar doméstico. ICONOFACTO, 9(12), 214-231. Recuperado de: https://dialnet.unirioja.es/descarga/articulo/5204342.pdf

Secretaría de Gobernación, (2014). Diario oficial de la federación. Recuperado de: http://dof.gob.mx/nota_detalle.php?codigo $=5342865 \&$ fecha $=30 / 04 / 2014$

Sola S. B. Capó, Vicedo, J. y Expósito L. M. (2005), Análisis de viabilidad de la aplicación de criterios de sostenibilidad en la construcción de edificios, IX Congreso de Ingeniería de Organización: Gijón, 8-9 septiembre de 2005. Valencia, España, Asociación para el Desarrollo de la Ingeniería de Organización.

Ullán de la Rosa, F. J. (2014). Sociología urbana: de Marx y Engels a las escuelas posmodernas. España: Centro de Investigaciones Sociológicas

Valdés, V. (2009) Producir o no producir: esa es la cuestión En: Ocaña, J., Portilla, M. y Jiménez, J. (coord.) El diseño ante el deterioro ambiental. Coloquio internacional de diseño 2008. México: Consejo Editorial de la Administración Pública Estatal.

WCED (1987). Our common future. The Brundtland Report. Oxford: Oxford University Press.

Ziccardi, A. (2015). Cómo viven los mexicanos. Análisis regional de las condiciones de habitabilidad de la vivienda. México: Universidad Nacional Autónoma de México, Instituto de Investigaciones Jurídicas. 
Ziccardi, A. y González, A. (2013). Política de vivienda y municipios. Memorial del Congreso Nacional de Vivienda 2013. México: Universidad Nacional Autónoma de México, Programa Universitario de Estudios sobre la Ciudad. Recuperado de: http://www.puec.unam.mx/site/cont_pdf/00_indice_general_relatoria.pdf

Zulaica, L. y Celemín, J. P. (2008). Análisis territorial de las condiciones de habitabilidad en el periurbano de la ciudad de Mar del Plata (Argentina), a partir de la construcción de un índice y de la aplicación de métodos de asociación espacial. Revista de Geografía Norte Grande, (41), 129-146. http://doi.org/10.4067/S0718-34022008000300007 